\title{
Measurement of Electric Field Distribution along the Plasma Column in Microwave Jet Discharges at Atmospheric Pressure
}

\author{
M. Abdur RAZZAK, Shuichi TAKAMURA, Takayuki TSUJIKAWA, Hideto SHIBATA \\ and Yuto HATAKEYAMA \\ Department of Electrical and Electronic Engineering, Faculty of Engineering, Aichi Institute of Technology, \\ Toyota 470-0392, Japan
}

(Received 27 July 2009 / Accepted 7 September 2009)

\begin{abstract}
A new technique for the direct measurement of electric field distribution along the plasma column in microwave jet discharges is developed and employed. The technique is based on a servomotor-controlled reciprocating antenna moving along the nozzle axis and plasma column. The measurement technique is applied to a rectangular waveguide-based $2.45 \mathrm{GHz}$ argon and helium plasma jets generated by using the modified TIAGO nozzle at atmospheric pressure with a microwave power of less than $500 \mathrm{~W}$. The measurement has been done with and without igniting the plasma jet in order to investigate the standing wave propagation along the nozzle axis and plasma column. It is observed that the electric field decay occurs slowly in space with plasma ignition than that of without plasma, which indicates the surface electromagnetic wave propagation along the plasma column in order to sustain the plasma jet. This study enables one to design, determine and optimize the size and structure of launcher nozzle, which plays an important role for the stable and efficient microwave plasma generators.
\end{abstract}

(C) 2009 The Japan Society of Plasma Science and Nuclear Fusion Research

Keywords: atmospheric pressure plasma, microwave jet discharge, electric field distribution, TIAGO nozzle

DOI: $10.1585 /$ pfr.4.047

\section{Introduction}

Atmospheric-pressure plasmas have attracted much interest recently for applications in materials synthesis, surface treatment, etching, film deposition, medical treatment, light sources, analytical chemistry, environmental applications and many more [1-3]. They may also have great potentiality for the very high heat-flux plasma beam intended for fundamental research on plasma-wall interactions (PWI) in next-generation fusion devices, such as ITER (International Thermonuclear Experimental Reactor) and DEMO (DEMOnstration Power Reactor) [4-6]. In this regard, the development of powerful, stable and efficient plasma generators is of high demand.

In recent years, the production of microwave plasma jets at atmospheric pressure $[7,8]$ using the recently developed TIAGO (Torche à Injection Axiale sur Guide d'Ondes, in French) nozzle [8] become very popular due to its simplicity, easy ignition, unnecessity of vacuum chamber and sustainment of discharge at the open air. But to generate such stable and efficient plasmas using the TIAGO system, the launcher nozzle should be properly designed and characterized by the electrical parameters of the generated plasmas. Among them, the electric field is an important parameter to be known in plasmas such as RF and microwave discharges since the electric field distribution in plasmas determines the energy distribution and homogeneity of the discharge. But in-situ measure- ment of microwave electric field is often difficult due to the lack of suitable measurement techniques. This is particularly true for the measurement of electric field in atmospheric microwave discharges. So far, spectroscopic techniques using laser-induced spectroscopy [9-13], fluorescence spectroscopy [14], optogalvanic spectroscopy [15], Doppler broadening [16,17] and Stark effects [18-23] are used to measure the electric field distribution in plasmas. Some other techniques such as electric field sensor [24-26], Raman scattering [27], electric field analyzer [28], neutral beam injection [29], non-linear optical tagging [30], millimeterwave reflectrometry [31] and electrostatic probe [32-34] are also used to investigate the electric field in various plasmas. However, until the present time any suitable direct measurements of electromagnetic fields in such an atmospheric microwave discharges are absent. Moreover, there are not so many techniques of microwave field investigation in free space, where microwave discharges take place.

From the above-mentioned motivation, in this work, a new technique for the direct measurement of electric field distribution in atmospheric microwave jet discharges along the nozzle axis and plasma column is developed and employed. The technique is based on a position controllable (controlled by servomotor) reciprocating antenna moving along the nozzle axis and plasma column. The measurement technique is applied to a rectangular waveguidebased $2.45 \mathrm{GHz}$ argon and helium plasmas at atmospheric 
pressure generated by using the TIAGO nozzle [8] with a slight modification in its design. The measurement has been done with and without igniting the plasma jet aiming to investigate the characteristics of the launcher nozzle, and to observe the standing wave propagation along the nozzle axis and plasma column.

\section{Microwave Plasma Production}

The schematic diagram of experimental setup for the generation of microwave plasma jets at atmospheric gas pressure is depicted in Fig. 1. The complete microwave circuit consists of a magnetron source $(1.5 \mathrm{~kW}, 2.45 \mathrm{GHz})$, WRJ-2 rectangular waveguide (combination of both flat and tapered), an isolator, a directional coupler, an E-H tuner, a short plunger and a coupling launcher. The E-H tuner is used in order to have no reflected power back to the source ensuring stable transfer of microwave power (less than $500 \mathrm{~W}$ in the present case) through the waveguide to the launcher nozzle by employing $\mathrm{TE}_{10}$ mode structure.

The strong electric field of $\mathrm{TE}_{10}$ mode enhances the intensity at the narrow gap section followed by the tapered waveguide. The short plunger placed at the microwave cir- cuit end produces the standing wave so that the electric field is increased (possibly the maximum) at the launcher position on the flat waveguide with a reduced height between H-planes. The short plunger is adjusted so that the distance between the short plunger and the launcher become an odd multiple number ( 9 or 11 in the present case) of a quarter wavelength of waveguide, $\lambda_{\mathrm{g}}$ (about $147.7 \mathrm{~mm}$ in the present case), which confirms the maximum microwave field at the launcher position.

We employed the modified TIAGO nozzle [8] for the launcher and gas injection for the microwave-sustained plasma jet. The original and modified TIAGO nozzles are depicted in Figs. 1 (b) and (c), respectively. The original TIAGO nozzle has an all along conical shape with a flat top. The modified TIAGO nozzle, on the other hand, is a combination of cylindrical and conical structure (for example, in case of nozzle with $80 \mathrm{~mm}$ length, $60 \mathrm{~mm}$ cylindrical and $20 \mathrm{~mm}$ conical) having a conical shape with sharp edge at the nozzle head. Because of conical structure of the launcher nozzle, the microwave field dissipating the microwave energy around the top is enhanced where the discharge occurs due to the field shaping characteristics of the

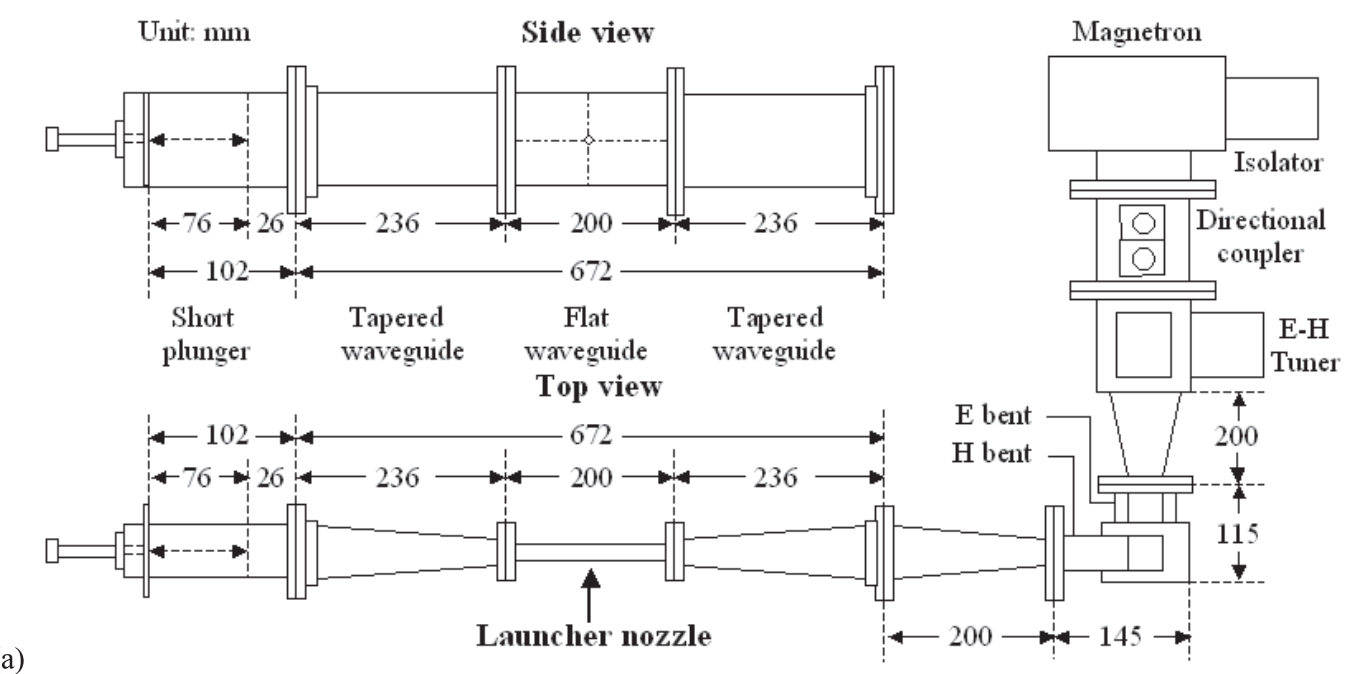

(a)

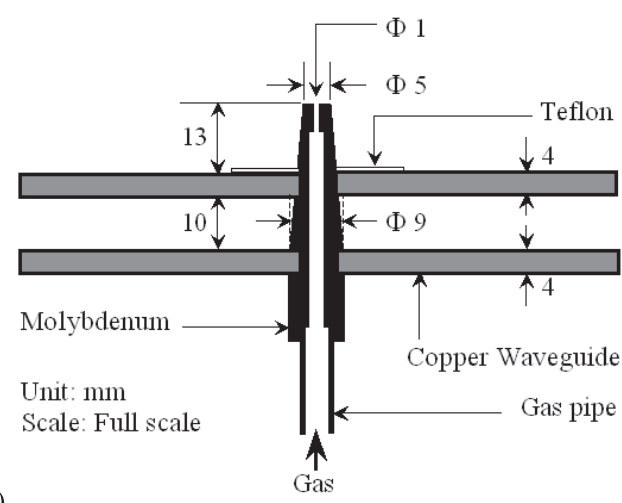

(b)

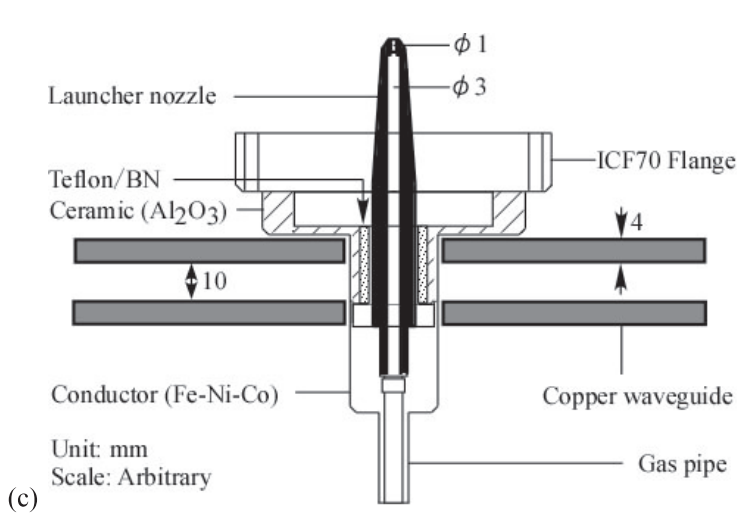

Fig. 1 Experimental device. (a) Microwave circuit for plasma jet discharge, (b) Original TIAGO nozzle set on the flat waveguide [8], (c) Sketch of modified TIAGO nozzle with ICF70 flange used in the present experiment for microwave-sustained plasma torch. 
nozzle. This feature is confirmed by the microwave electric field simulation that ensures the increased electric field intensity around the top of the launcher nozzle as shown in Fig. 2. The simulation results also validate the modification of TIAGO nozzle used in the present experiments for producing the microwave-induced plasma jets. The de-
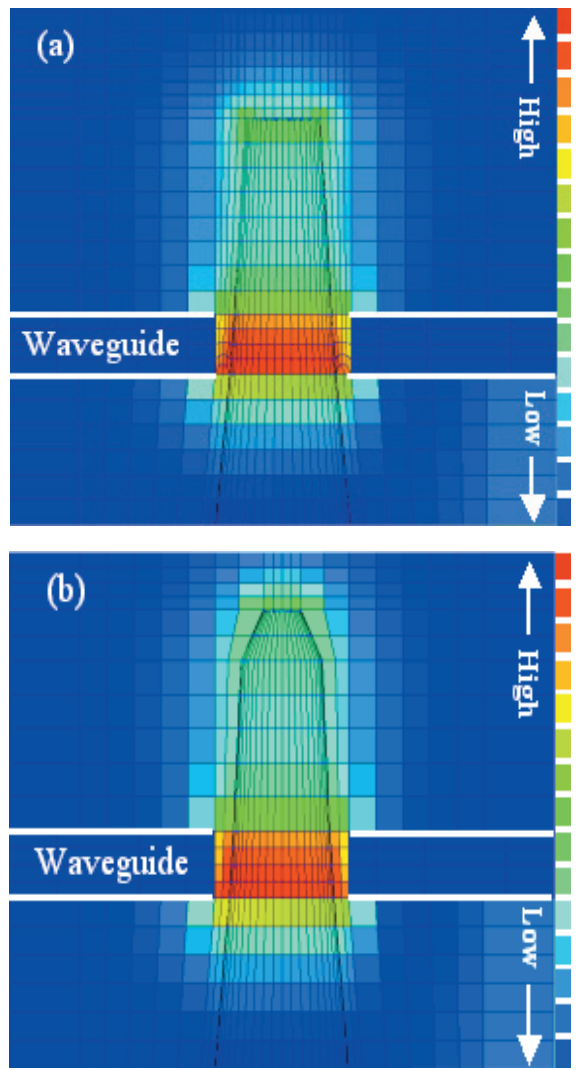

Fig. 2 Simulation results of microwave electric field distribution for (a) original TIAGO nozzle and (b) modified TIAGO nozzle.

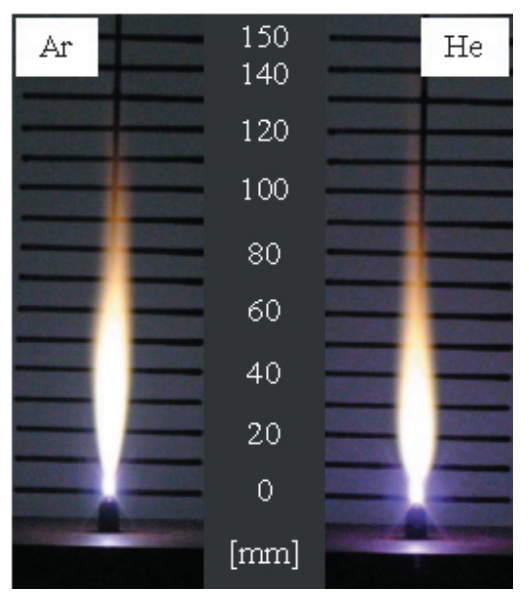

Fig. 3 Typical still photo of argon $(0.5 \mathrm{~L} / \mathrm{min})$ and helium $(1.0 \mathrm{~L} / \mathrm{min})$ plasma jets with spindle shape. Microwave input power for both cases is $320 \mathrm{~W}$. tails of the simulation of microwave electric field will be discussed elsewhere.

Argon and helium are used as working gases with a flow rate of $0.5 \sim 1.5 \mathrm{~L} / \mathrm{min}$ and a microwave power less than $500 \mathrm{~W}$. The initial ignition of the discharge is assisted by a Tesla coil. The flame-like plasma starting from the head of the launcher nozzle is formed according to the field distribution radiated from the waveguide hole. Typical still photos of argon and helium plasma torches corresponding to a so-called normal straight discharge with a spindle shape, one of the bifurcated discharge modes [7], are shown in Fig. 3.

\section{Measurement of Electric Field}

The schematic diagram for the measuring system of microwave electric field is shown in Fig.4(a), which is composed of a servomotor, a tungsten-made pick-up antenna ( $L=7 \mathrm{~mm}, \phi=0.2 \mathrm{~mm})$ covered by a uniform tube having a characteristic impedance of $50 \Omega$, a diode (HP 423B), an attenuator ( $30 \mathrm{~dB})$, and an A-D converter followed by a personal computer for data acquisition. One end of antenna is made to bend $90^{\circ}$ to make the coaxial tube an L-shape in order to avoid the possible damage while the other side is ended with a TNC connector to connect it with the diode and other devices as shown

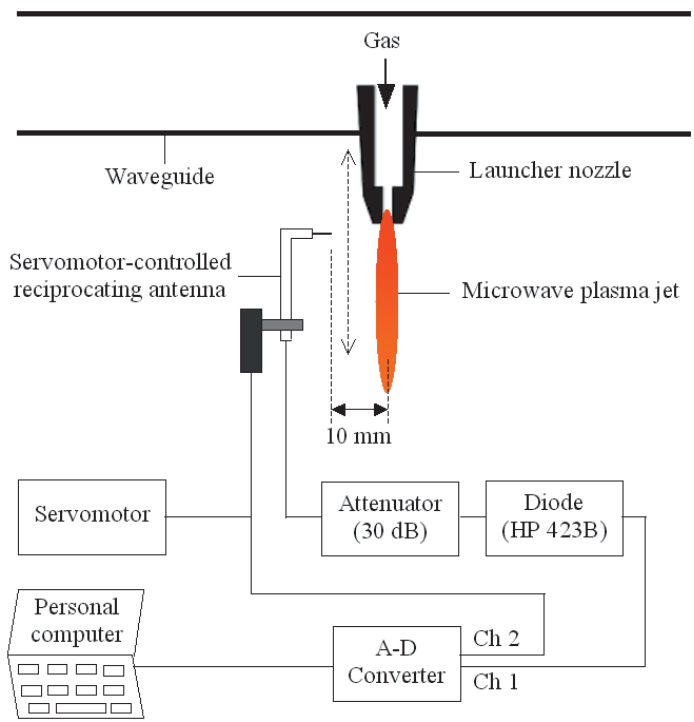

(a)

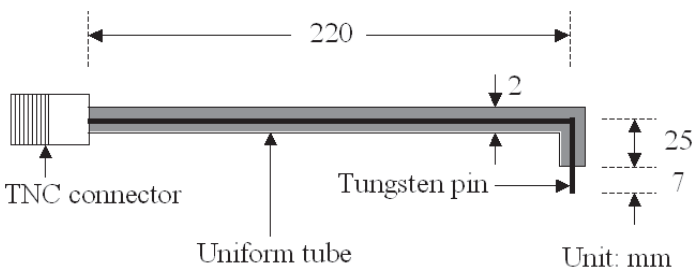

(b)

Fig. 4 Electric field measuring system. (a) Microwave electric field measuring circuit, (b) Sketch of tungsten antenna. 


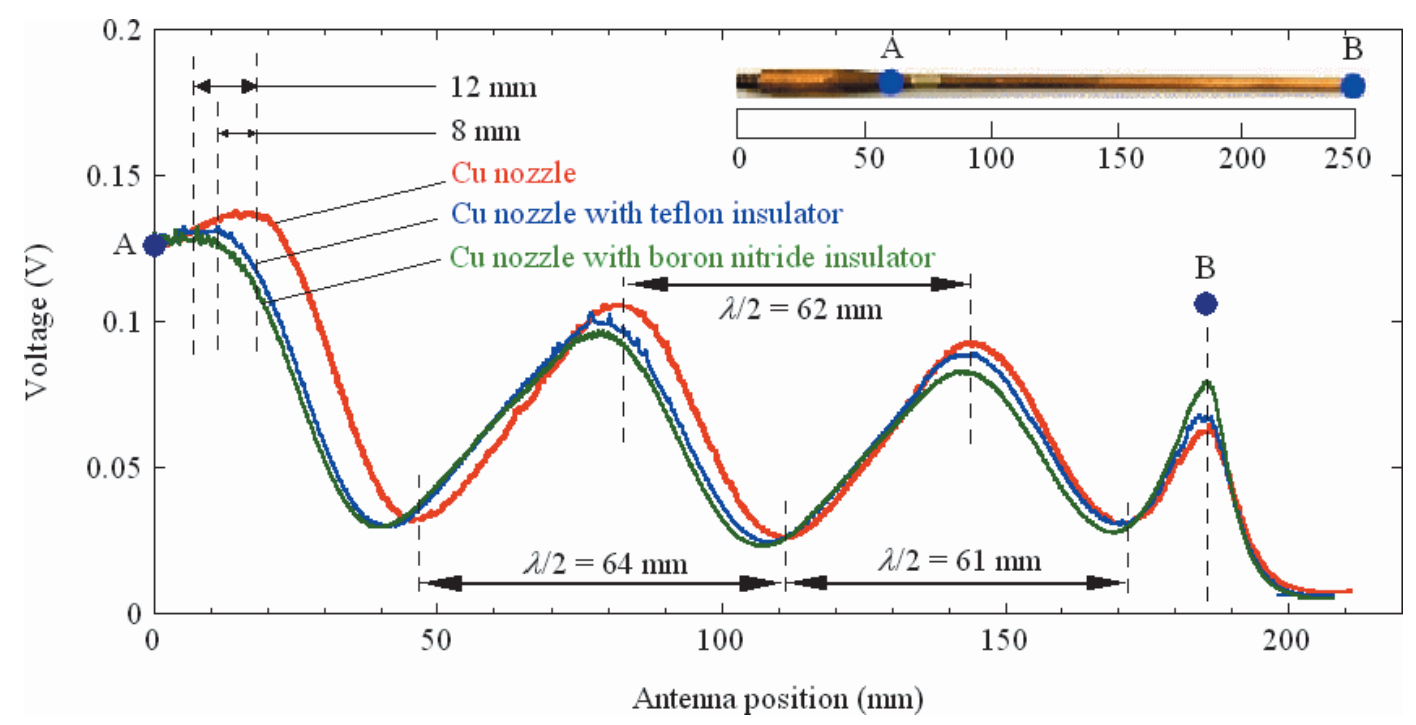

Fig. 5 Electric field distribution along the nozzle axis with a $250 \mathrm{~mm}$ long Cu nozzle (test launcher) without igniting the plasma. Electric field distributions using $250 \mathrm{~mm}$ long $\mathrm{Cu}$ nozzle with Teflon and boron nitride insulator spacer adjusting at the waveguide end are also plotted for comparison.

in Fig. 4 (b). The antenna tip of the bend portion is adjusted about $10 \mathrm{~mm}$ from the nozzle axis and center of the plasma column to avoid the direct contact of antenna with the launcher nozzle and plasma jet, which may cause burn and damage of the antenna and/or diode. The reciprocating motion of antenna is controlled by the servomotor to move back and forth along the nozzle axis and plasma column as shown in Fig. 4 (a). The servomotor, which is powered by a dc source, is operated manually. When the servomotor is switched on, the antenna moves freely back and forth along the direction of nozzle axis and plasma column. The device is designed by using a relay system in such a way that, when moving toward the waveguide end where the nozzle is connected, the antenna will be stopped automatically $2.5 \mathrm{~mm}$ away from that end and will also be stopped automatically about $220 \mathrm{~mm}$ away from the waveguide end when moving outward from that end in order to avoid the possible damage of the device. The antenna is connected to an A-D converter (WE7000 Ethernet) followed by a diode (Model: HP 423B, 0.1 12.4 GHz, $\pm 3 \mathrm{~dB}$ ). An attenuator of $30 \mathrm{~dB}$ is placed in front of diode in order to avoid the possible damage of the diode. The antenna position is recorded by using a potentiometer controlled by the servomotor. In the A-D converter two separate channels are used: one is used for measuring the antenna voltage while the other is used for recording the antenna position. Finally the data are stored in a personal computer for analysis.

\section{Results and Discussions}

The microwave electric field distribution is investigated by different type of nozzles, in terms of size, structure and materials, with and without igniting the plasma in order to investigate the electrical characteristic of noz- zle, which is practically helpful to have the proper choice of launcher nozzle. At first, we have investigated the electric field distribution with a $250 \mathrm{~mm}$ long solid $\mathrm{Cu}$ nozzle (test launcher) without igniting the plasma in order to understand the microwave (standing wave) propagation along the nozzle axis. Then, we have investigated the electric field distribution with the nozzles made by three different materials e.g. molybdenum $(\mathrm{Mo})$, copper $(\mathrm{Cu})$ and $\mathrm{Mo}$ with silver $(\mathrm{Ag})$ coating with a length varying from 65 to $110 \mathrm{~mm}$ and a diameter of $6.3 \sim 8.8 \mathrm{~mm}$ at the starting end. The starting position of the measurement is about $62 \mathrm{~mm}$ away from the nozzle end connected to the waveguide and about $2.5 \mathrm{~mm}$ away from the waveguide end.

Figure 5 shows the electric field distribution along the nozzle axis for a $250-\mathrm{mm}$ long solid $\mathrm{Cu}$ nozzle (test launcher). The positions of the measurement starting point and that of the nozzle head (end) are indicated by points $\mathrm{A}$ and $\mathrm{B}$, respectively. It is seen that the microwave field radiating in space from the solid $\mathrm{Cu}$ nozzle (test launcher) is propagating along the nozzle axis with an average wavelength found to be approximately $61 \mathrm{~mm}$, about a half of the conventional wavelength of free space $(122.36 \mathrm{~mm})$ with a microwave frequency of $2.45 \mathrm{GHz}$, ensuring the standing wave propagation along the nozzle axis. It is observed that the amplitude of standing wave decreases with distance it travels along the nozzle axis due to the attenuation and damping factor of lossy copper medium, and radiation to the free space. It is also observed that the first peak of electric field occurs at about $18 \mathrm{~mm}$ from the measurement starting point or about $80 \mathrm{~mm}$ from the nozzle end connecting at the waveguide, which enables us to pick the right choice of nozzle length of about $80 \mathrm{~mm}$ long in the present experimental conditions.

One of our research goals is to inject the plasma jet 
into an airtight chamber having pure working gas environment in order to study the plasma-wall interaction (PWI). For this purpose, in the present experimental setup, the launcher system for microwave-sustained plasma torch injecting into the chamber is designed accordingly by connecting the modified TIAGO nozzle with ICF70 flange as shown in Fig. 1(c). But some spurious discharge is observed around the launcher nozzle connecting at the waveguide end due to the presence of some space between the waveguide hole and launcher nozzle. Insulators like Teflon or boron nitride can be used to the launcher nozzle end connecting with the waveguide in order to solve the problem avoiding the gas space and consequently the spurious discharge. However, in order to use such an insulator, the size of the launcher nozzle must be determined by analyzing the electric field distribution since it is assumed that Teflon or boron nitride insulator may have some effect on electric field propagation. Therefore, the electric field distributions using 250-mm long $\mathrm{Cu}$ nozzle with Teflon $(L=25 \mathrm{~mm}$, inner diameter $=6.35 \mathrm{~mm}$, outer diameter $=8.35)$ and boron nitride $(L=30 \mathrm{~mm}$, inner diameter $=8.35 \mathrm{~mm}$, outer diameter $=6.6 \mathrm{~mm}$ ) insulators at the waveguide end are also investigated and plotted in Fig. 5 for comparison. It is observed that the microwave propagation characteristic has been changed near the insulator by using the insulator spacers at the waveguide end. It is seen that, comparing with the $\mathrm{Cu}$ nozzle, the first peak of electric field is shifted to left of about $8 \mathrm{~mm}$ and $12 \mathrm{~mm}$, respectively when using the Teflon and boron nitride insulator at the launcher nozzle end connecting with the waveguide. This feature comes from the different dielectric constants of Teflon (2.1) and that of boron nitride (4.6). This analysis provides us the length of the launcher nozzle of about $72 \mathrm{~mm}$ and $68 \mathrm{~mm}$, respectively, while using the Teflon and boron nitride insulator with $\mathrm{Cu}$ nozzle in the present case for efficient generation the microwave plasma jets.

Figure 6 shows the comparison of electric field distribution along the nozzle axis for $80-\mathrm{mm}$ long launcher nozzles made by $\mathrm{Cu}$, Mo and Mo with $\mathrm{Ag}$ coating without igniting the plasma. Like simulation results shown in Fig. 2, it is observed that the microwave electric field along the nozzle axis is enhanced around the nozzle head for all kind of nozzle, which is helpful for the easy ignition and sustainment of microwave-induced plasma jets. It is noticed that, for the nozzle made by Mo with Ag coating, the electric field increases sharply along the nozzle axis, the peak is shifted more away from the measurement starting point and is found to be higher than those measured by the $\mathrm{Cu}$ and Mo nozzles of same length. These features indicate better propagation of microwave field using the Mo nozzle with Ag coating due to the very small skin depth of Ag. We have also done the same experiments with nozzles of various lengths of $65,68,70,72,75$ and $100 \mathrm{~mm}$ made by $\mathrm{Cu}$ and Mo. It is observed that the material of launcher nozzle does have no such significant influence for the microwave propagation rather it is influenced by the struc-

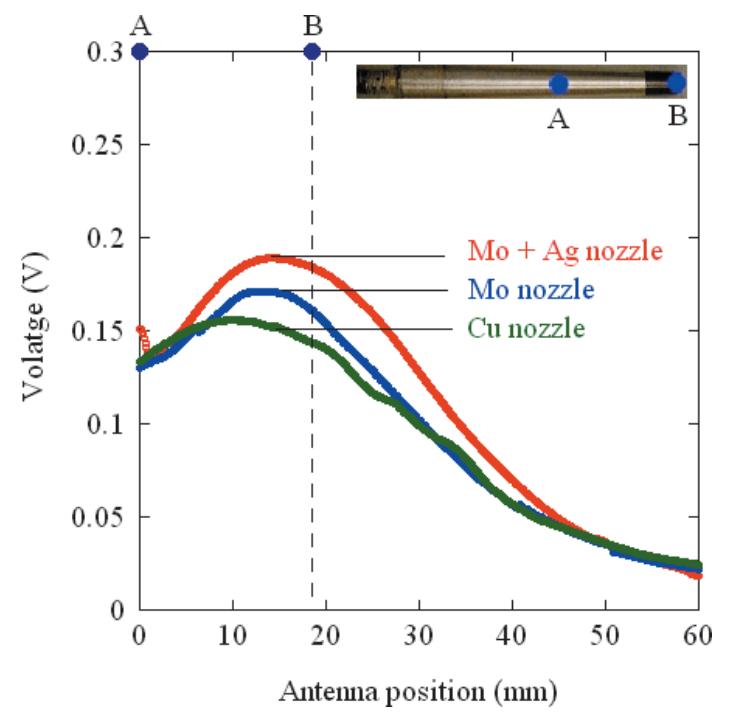

Fig. 6 Electric field distribution along the nozzle axis without igniting the plasma with 80 -mm long nozzles made by $\mathrm{Cu}$, Mo and Mo with Ag coating.

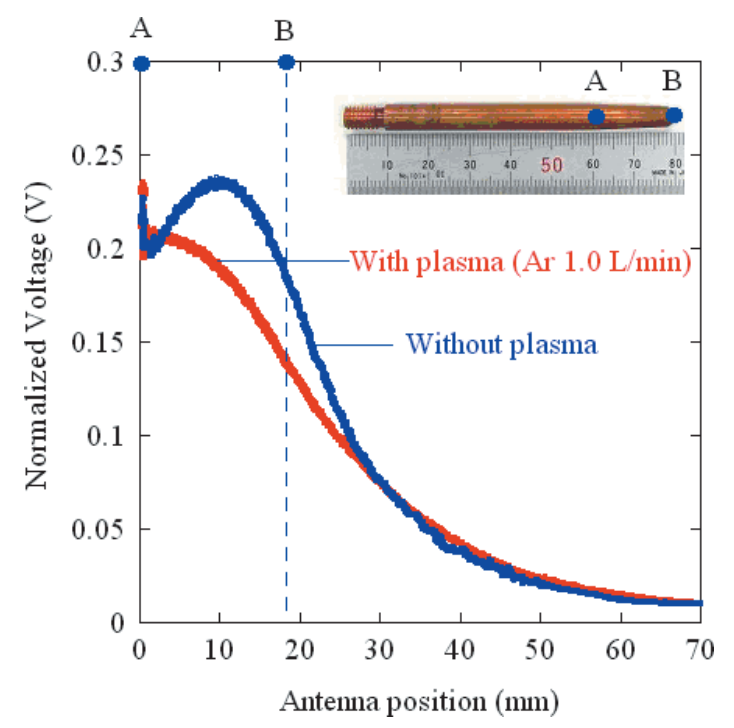

Fig. 7 Electric field distribution along the nozzle axis with a 80-mm Cu nozzle with and without igniting the plasma.

ture and size. From our investigations, the launcher nozzle having a length of $80 \mathrm{~mm}$ and a diameter of $6.5 \mathrm{~mm}$ at the starting end is found to be the best suited for the generation of microwave-induced plasma jet under the present experimental conditions. Therefore, we have chosen $\mathrm{Cu}$ nozzle of $6.5 \mathrm{~mm}$ diameter and $80 \mathrm{~mm}$ length, with $60 \mathrm{~mm}$ cylindrical and $20 \mathrm{~mm}$ conical in shape, as shown in Fig. 1 (c) for further investigations since $\mathrm{Cu}$ is less expensive than Mo.

Then, we have investigated the microwave electric field distribution along the nozzle axis with a 80-mm long $\mathrm{Cu}$ nozzle by applying $\mathrm{Ar}$ and $\mathrm{He}$ as the working gas with a flow rate of $0.5 \sim 1.6 \mathrm{~L} / \mathrm{min}$ and a microwave input power 

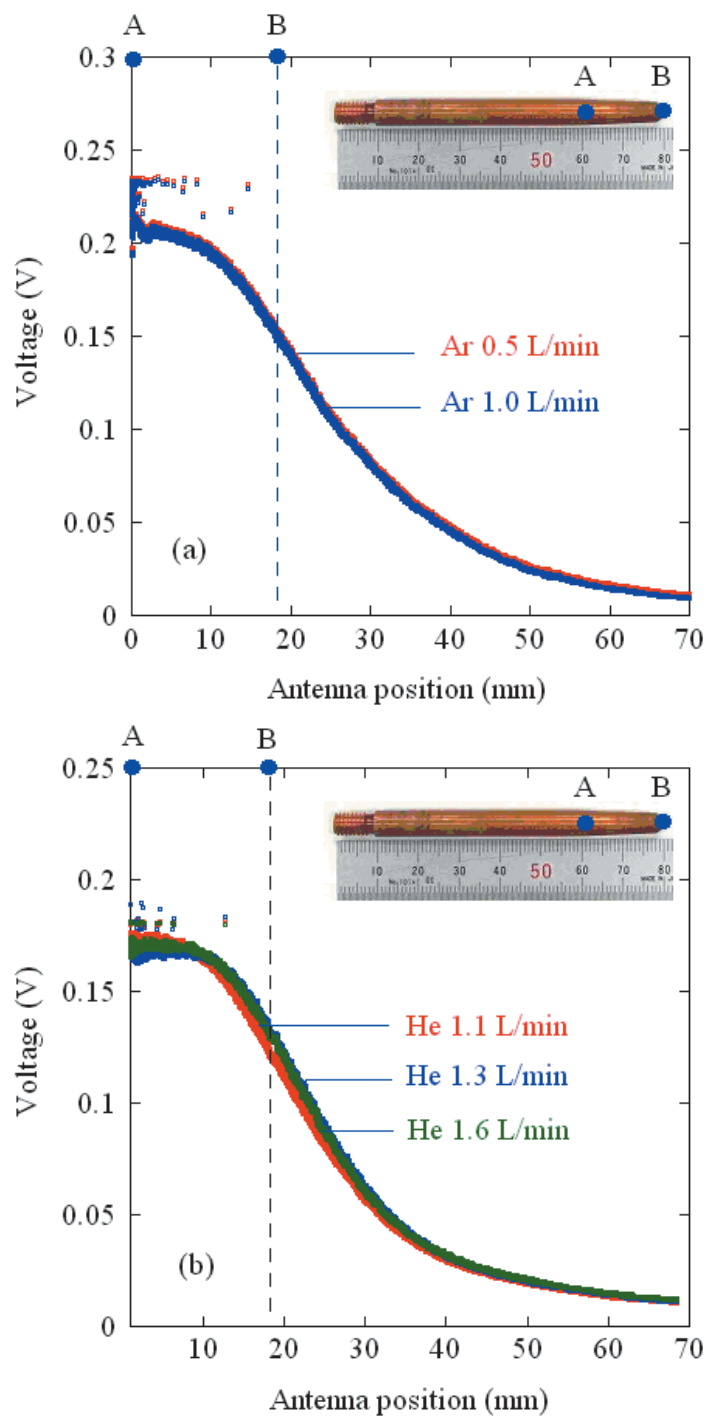

Fig. 8 Electric field distribution along the nozzle axis with a 80-mm Cu nozzle for various gas flow rates. (a) Comparison between Ar plasmas (b) Comparison among $\mathrm{He}$ plasmas. Microwave input power is $320 \mathrm{~W}$.

of about $320 \mathrm{~W}$ for both the gas species. The investigation has been done both with and without igniting the plasma. Figure 7 shows the comparison of microwave electric field distribution along the nozzle axis and plasma column with and without igniting the plasma. It is observed that the electric field is enhanced along the nozzle axis for a short period when the plasma is not ignited before starting to decay. On the other hand, the electric field starts to decrease from the beginning with the plasma ignition due to plasma loading, which ensures the surface electromagnetic wave propagation along the plasma column in order to sustain the plasma. It is also noticed that the electric field decay occurs slowly in space when the plasma is ignited which may be an indication that the microwave electric field propagates not only with the launcher nozzle but also with the plasma column that helps to sustain the stable mi-

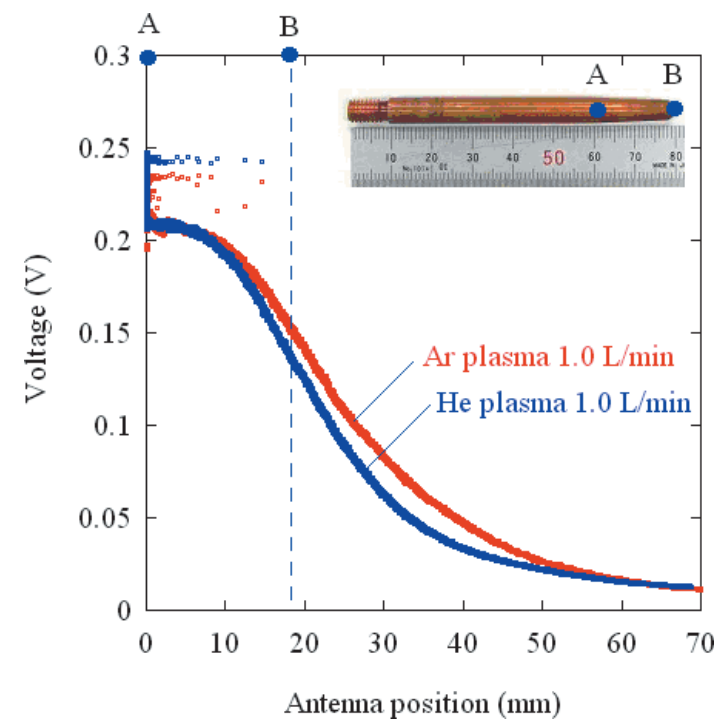

Fig. 9 Comparison of electric field distribution in $\mathrm{Ar}$ and $\mathrm{He}$ plasmas with a microwave power of $320 \mathrm{~W}$.

crowave plasma jets. It is noticed that although the plasma flame observed in the open air is about $80 \sim 100 \mathrm{~mm}$ long as shown in Fig. 3, it is observed that the intensity of the flame decreases sharply after the height of 50 or $60 \mathrm{~mm}$. Since the field intensity is measured in space about $10 \mathrm{~mm}$ away from the center of the plasma flame, a weaker electric field intensity is observed after the $30 \sim 40 \mathrm{~mm}$ from the nozzle head as shown in Figs. 6 9.

Figures 8 (a) and (b) show the comparison of microwave electric field distribution along the nozzle axis for argon and helium plasmas, respectively, with various gas flow rates and a fixed microwave input power of about $320 \mathrm{~W}$. In both cases it is seen that the curves of electric field distribution overlap among themselves indicating that the gas flow rate has no such significant influence on the electric field distribution with a fixed microwave input power.

The electric field distribution for Ar and He plasmas is compared in Fig. 9. The electric field with faster decay is found in case of He plasma than that of Ar one with the same gas flow rate and microwave input power indicating Ar plasmas seems to be more conductive than the He ones. It also indicates an easier ignition and generation of microwave-induced Ar plasma jet than that of He one with such a modified TIAGO nozzle.

Finally, the plasma parameters (electron temperature and electron density) are estimated by using both optical emission spectroscopy (OES) and Langmuir probe measurement technique. The electron temperature of plasma is found to be in the order of $10^{4} \mathrm{~K}$ while the electron density found in the order of $10^{19} \mathrm{~m}^{-3}$. 


\section{Summary}

In this paper, the development of a new technique for the direct measurement of electric field distribution in a microwave discharge along the nozzle axis and plasma column is reported. The technique is based on a servomotorcontrolled tungsten-made pick-up antenna moving along the nozzle axis and plasma column. The measurement technique is applied to a rectangular waveguide-based $2.45 \mathrm{GHz}$ argon and helium plasmas at atmospheric pressure with a microwave power of less than $500 \mathrm{~W}$ generated by using the modified TIAGO nozzle. This study will certainly help one to design the launcher nozzle properly by optimizing its size and structure. The measurement has been done with and without igniting the plasma jet at atmospheric pressure. It is found that the peak value of electric field is smaller and has delayed decay after the nozzle head with plasma ignition than that of without plasma, which confirms the surface electromagnetic wave propagation along the plasma column in order to sustain the stable microwave plasma jets. The electron temperature and density of the generated plasmas are found in the order of $10^{4} \mathrm{~K}$ and $10^{19} \mathrm{~m}^{-3}$, respectively.

\section{Acknowledgement}

This work is supported by Grant-in Aid received from the Japan Society for the Promotion of Science (No. 2008409).

[1] K. Yamakawa et al., J. Appl. Phys. 98, 013301 (2005).

[2] S.J. Kyung et al., J. Appl. Phys. 100, 124303 (2006).
[3] M. Laroussi et al., Appl. Phys. Lett. 87, 113902 (2005).

[4] S. Ferri et al., AIP Conf. Proc. 1058, 216 (2008).

[5] V.I. Afanasyev et al., AIP Conf. Proc. 988, 177 (2008).

[6] P.D. Maio et al., AIP Conf. Proc. 513, 274 (2000).

[7] S. Takamura et al., Plasma Fusion Res. 3, 012 (2008).

[8] M. Moisan et al., Plasma Sources Sci. Technol. 10, 387 (2001).

[9] K. Takiyama et al., Rev. Sci. Instrum. 59, 2351 (1988).

[10] M. Watanabe et al., Rev. Sci. Instrum. 70, 903 (1999).

[11] T. Kampschulte et al., New J. Phys. 9, 18 (2007).

[12] Y.W. Choi et al., Appl. Phys. Lett. 69, 1361 (1996).

[13] J.P. Booth et al., Appl. Phys. Lett. 65, 819 (1994).

[14] B.N. Ganguly et al., Plasma Sources Sci. Technol. 9, 437 (2000).

[15] C. Pérez et al., AIP Conf. Proc. 1058, 15 (2008).

[16] T. Kobayashi et al., Rev. Sci. Instrum. 75, 4121 (2004).

[17] A. Demura et al., AIP Conf. Proc. 645, 79 (2002).

[18] S. Reyes Cortes et al., Rev. Sci. Instrum. 74, 1596 (2003).

[19] A. Steiger et al., AIP Conf. Proc. 645, 86 (2002).

[20] E.K. Cherkasova et al., AIP Conf. Proc. 645, 413 (2002).

[21] K. Kamiya et al., Rev. Sci. Instrum. 72, 2931 (2001).

[22] V.P. Gavrilenko et al., Phys. Rev. E 62, 7201 (2000).

[23] B.W. Rice et al., Rev. Sci. Instrum. 70, 815 (1999).

[24] Y. Lee et al., Rev. Sci. Instrum. 68, 4427 (1997).

[25] A. Kumada et al., J. Appl. Phys. 84, 3059 (1998).

[26] G. Roussy et al., Meas. Sci. Technol. 11, 1145 (2000).

[27] T. Ito et al., J. Phys. D: Appl. Phys. 42, 092003 (2009).

[28] C. Charles et al., Phys. Plasmas 7, 5232 (2000).

[29] V.I. Davydenko et al., Plasma Phys. Control. Fusion 36, 1805 (1994).

[30] N. Claire et al., Rev. Sci. Instrum. 72, 4372 (2001).

[31] J.H. Lee et al., Rev. Sci. Instrum. 68, 462 (1997).

[32] S.V. Ratynskaia et al., Phys. Plasmas 9, 4135 (2002).

[33] R. Schrittwieser et al., AIP Conf. Proc. 669, 211 (2003).

[34] P. Pribyl et al., Rev. Sci. Instrum. 77, 073504 (2006). 